\title{
A Comparative Study on the Parametrization of a Block-based Compressive Sensing Algorithm for Hyperspectral Imaging Applications
}

\author{
Fernando Arias*†, Maytee Zambrano, Ph.D.*, Heidy Sierra, Ph.D. $\ddagger$ and Emmanuel Arzuaga, Ph.D. $\dagger$ \\ †Universidad de Puerto Rico, Recinto Universitario de Mayaguez, fernando.arias@upr.edu, earzuaga@ece.uprm.edu \\ *Universidad Tecnologica de Panama, maytee.zambrano@utp.ac.pa \\ \$Memorial Sloan Kettering Cancer Center, sierragh@mskcc.org
}

\begin{abstract}
Compressive Sensing-based technologies have shown a great potential to improve the efficiency of acquisition, manipu-lation, analysis and storage processes on signals and imagery with little discernible loss in data performance. The CS framework is based on the assumption that signals are sparse in some domain and can be reconstructed from a significantly reduced amount of samples. As a result, a solution to the underdetermined linear system resulting from this paradigm makes it possible to estimate the original signal with high accuracy using linear programming techniques. This paper presents a study on the use of compressive sensing on satellite Hyperspectral Images, which provide a variety of fields and applications with data with a high information density for analysis. Hyperspectral imaging of large areas at high resolutions required for some applications can turn the image capturing, processing and storage processes into a time consuming procedure, presenting a limitation for use in resource-limited or time-sensitive settings.

We present an analysis on the algorithm parametrization that may allow for a simpler capturing approach tailored specifically for a given application's needs using the well-studied l1-magic algorithm. We provide a comparative study in compressive sensing and estimate its effectiveness in terms of compression ratio vs. image reconstruction accuracy. Preliminary results show that by using as little as $25 \%$ of the original number of samples, large structures may be reconstructed with high accuracy.
\end{abstract}

Digital Object Identifier

(DOI):http://dx.doi.org/10.18687/LACCEI2016.1.1.310

ISBN: 978-0-9822896-9-3

ISSN: 2414-6390

$14^{\text {th }}$ LACCEI International Multi-Conference for Engineering, Education, and Technology: "Engineering Innovations for Global Sustainability", 20-22 July 2016, San José, Costa Rica. 


\title{
A Comparative Study on the Parametrization of a Block-based Compressive Sensing Algorithm for Hyperspectral Imaging Applications
}

\author{
Fernando Arias* ${ }^{* \dagger}$, Maytee Zambrano, Ph.D.*, Heidy Sierra, Ph.D. ${ }^{\ddagger}$ and Emmanuel Arzuaga, Ph.D. ${ }^{\dagger}$ \\ †Universidad de Puerto Rico, Recinto Universitario de Mayaguez, fernando.arias@upr.edu, earzuaga@ece.uprm.edu \\ *Universidad Tecnologica de Panama, maytee.zambrano@utp.ac.pa \\ ${ }^{\ddagger}$ Memorial Sloan Kettering Cancer Center, sierragh@mskcc.org
}

\begin{abstract}
Compressive Sensing-based technologies have shown a great potential to improve the efficiency of acquisition, manipulation, analysis and storage processes on signals and imagery with little discernible loss in data performance. The CS framework is based on the assumption that signals are sparse in some domain and can be reconstructed from a significantly reduced amount of samples. As a result, a solution to the underdetermined linear system resulting from this paradigm makes it possible to estimate the original signal with high accuracy using linear programming techniques. This paper presents a study on the use of compressive sensing on satellite Hyperspectral Images, which provide a variety of fields and applications with data with a high information density for analysis. Hyperspectral imaging of large areas at high resolutions required for some applications can turn the image capturing, processing and storage processes into a time consuming procedure, presenting a limitation for use in resourcelimited or time-sensitive settings.

We present an analysis on the algorithm parametrization that may allow for a simpler capturing approach tailored specifically for a given application's needs using the well-studied l1-magic algorithm. We provide a comparative study in compressive sensing and estimate its effectiveness in terms of compression ratio vs. image reconstruction accuracy. Preliminary results show that by using as little as $\mathbf{2 5 \%}$ of the original number of samples, large structures may be reconstructed with high accuracy.
\end{abstract}

\section{INTRODUCTION}

Hyperspectral imaging devices provide the ability to observe radiance or reflectance intensity across hundreds of spectral bands. Hyperspectral images are widely used in a variety of environmental, agricultural, military, geological, biological and surveillance applications due to the large information volume they contain. Hyperspectral images are dimensioned as a series of two-dimensional images in the spatial domain, with an additional spectral dimensional component. Useful as they are, hyperspectral images have the important cost of a larger data size, which has consequences in storage requirements, aquisition time, system complexity and bandwidth requirements for transmission of acquired images. These reasons justify why a variety of applications operate on a use it or lose it philosophy, where recently acquired hyperspectral data is processed for relevant information, and then discarded due to the unfeasibility of long-term storage of large amounts of hyperspectral data.

Compressive Sensing (from here on, referred to only as $C S$ ) is a signal processing framework for efficiently sensing and reconstructing signals at a sub-Nyquist rate. Unlike the conventional methodology in signal processing, where the entirety of the information available to a detector is collected and then compressed following the Shannon-Nyquist sampling theorem, CS reconstructs the original signal through solving an optimization problem from a relatively small amount of measurements. CS is based on the knowledge that a small collection of linear projections of a sparse signal in some domain contains enough information for its recovery. By following this alternate approach, there is a potentially large reduction in the sampling and computation requirements of a signal detection or estimation system using CS, which translates into a proportional large reduction of energy, bandwidth, and processing time requirements for a given application [1] [2]. CS is based on the work of Candès, Romberg and Tao [3] and Donoho [4], who showed that a signal having a sparse representation in some domain can be reconstructed from a small set of non-adaptive, linear measurements if they meet some specific criteria. CS can be used, not only for signal reconstruction or approximation, but also in making a detection or classification decision or estimation, which makes the CS technique a very powerful approach to many applications and to a wide range of statistical inference tasks [5].

Previous work on the use of CS technology in hyperspectral imaging applications has made advancements using a variety of approaches such as coded apertures [6][7] and spectral unmixing [8]. In this paper, we perform a preliminary comparative study of the various acquisition parameters available to us on real hyperspectral imagery data, and their impact on the quality of the resulting reconstructed images. We aim to demonstrate that CS is a viable solution to reducing the temporal and computational resource requirements of hyperspectral image acquisition, and provide a referential guideline for determining the individual best CS parametrization for a given application. A CS approach considerably reduces the required sensor usage time by reducing the amount of required samples and, consequently, the ability to capture a significantly larger amount of samples in a given amount of time.

The rest of this document is organized as follows: Section III describes the data used for evaluation and the various processing procedures performed on our data. Section IV

Digital Object Identifier (DOI): http://dx.doi.org/10.18687/LACCEI2016.1.1.310 ISBN: 978-0-9822896-9-3

ISSN: $2414-6390$

14th LACCEI International Multi-Conference for Engineering, Education, and Technology: "Engineering Innovations for Global Sustainability", 20-22 July 2016, San José, Costa Rica. 
presents the results from the procedures performed on Section III. Finally, the results of the aforementioned procedures are discussed, and main conclusions of our analysis and future work are presented in Section V.

\section{HYPERSPECTRAL IMAGING VIA COMPRESSIVE SENSING}

To illustrate the concept of CS, suppose that we have an $\mathrm{N}$ dimensional vector signal $x \in R^{N}$, which can be sparsely represented as $s$ in an alternate domain by the transform matrix $\psi$. In this alternate representation, $x$ and $s$ are both valid representations of the same signal, with $x$ existing in the spatial domain, and $s$ existing in the $\psi$ domain. If this signal takes a relatively small amount of $K$ nonzero values in any domain $\psi$, we can conclude that the signal is $K$-sparse in this domain.

CS theory proposes that if $x$ is sparse, it could be accurately recovered to a high degree of accuracy by taking $m$ random measurements of $x$, where $m$ is much smaller than $N$ [3]. In order to take the measurements, steps must be taken to produce a random sampling matrix $A$ of size $N \times m$, where $m \ll N$. Thus, the compressively sampled signal, $y$, is given by the following equation:

$$
y=\mathbf{A} x
$$

The compressively sampled signal $\hat{y}$ can be reconstructed from the incompletely sampled information at any moment by solving the following optimization problem:

$$
\hat{y}=\operatorname{argmin}\left\|\psi^{T} x\right\|_{k} \quad \text { s.t. } \quad y=\mathbf{A} x
$$

There are a wide variety of available algorithms suited to solving this optimization problem, in the context of reconstructing compressively sensed signals. A comparative analysis of commonly used algorithms for this task has been performed by Nunez et al [9]. According to the results in [9], we chose the min-TV algorithm available in the L1Magic package [10] for the reconstruction from compressive samples of our imagery, which minimizes the total-variation norm of an object. It is common for reconstruction algorithms used for signal reconstruction in CS applications to return an approximate reconstruction by minimizing the 11 norm using a variety of methods. However, approaches using the TV norm have provided us with faster and more accurate reconstructions, making it ideal for a reconstruction algorithm for hyperspectral imaging applications. Total-variation norm minimization seeks, through a convex optimization problem, to solve the problem of returning an approximation $\hat{y}$ of the original signal $x$ through an optimization problem that minimizes the TV norm:

$$
\|\hat{y}\|_{T V}=\sum_{t 1, t 2} \sqrt{\left|D_{1} \hat{y}(t 1, t 2)\right|^{2}+\left|D_{2} \hat{y}(t 1, t 2)\right|^{2}}
$$

where $D_{1}$ is the difference given by $D_{1} \hat{y}=\hat{y}(t 1, t 2)-\hat{y}(t 1-$ $1, t 2)$ and, similarly, $D_{2} \hat{y}=\hat{y}(t 1, t 2)-\hat{y}(t 1, t 2-1)$. This procedure is further illustrated in [3]. Further, we have developed an algorithm which separates this optimization problem into a large set of smaller, faster, independent routines to provide reasonable reconstruction times for large images. This procedure is outlined in Section II-A. By reducing aquisition time and complexity in a Hyperspectral imaging system, it is possible to acquire considerably more hyperspectral data in a given amount of time, while maximizing the efficiency of bandwidth and storage resources. Futhermore, it opens the possibility of long-term storage of compressively sampled hyperspectral images in applications where storage of conventionally sample data is unfeasible or unjustifiable.

\section{A. Block-Compressive Sensing Algorithm}

In order to increase computational efficiency and attain reasonable reconstruction times for large resolution images (either spatially or spectrally), a block-based Compressive Sensing reconstruction approach was devised. The proposed approach divides hyperspectral data cubes from a hyperspectral imaging device into a set of individual two-dimensional spectral bands. Following this, each individual spectral band image is divided into smaller blocks, which are independently reconstructed from compressive samples and assembled into a complete 3$\mathrm{D}$ data cube with a high degree of precision. This procedure is visually outlined in Fig. 1. In the event of having a single band as the input, such as the case of the datasets evaluated in this work, the first splitting step is skipped, and the spectral band in question divided into blocks immediately.

In order to meet the variety of accuracy and/or reconstruction time constraints present in a variety of hyperspectral imaging applications (as well as other fields that share similar limitations in handling and processing data with a similar mathematical structure), both the block size and the sampling factor, which indicates the percentage of compressively sampled points in a given block, may be freely modified to achieve an acceptable tradeoff between reconstruction accuracy and total reconstruction times and determine the influence of each of these parameters on the reconstructed imagery.
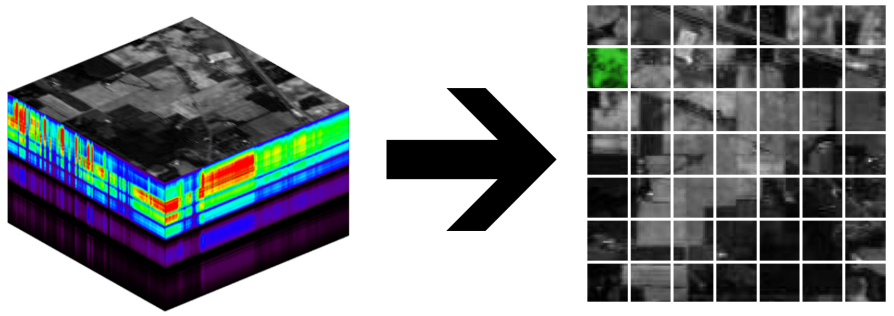

Fig. 1: Operation description for the block-CS algorithm. With a 3-D hyperspectral cube as the input, the algorithm separates the image into bands, and individual bands into blocks. The individual blocks (highlighted in green) are for illustrative purposes and not shown to any specific scale.

\section{EXPERIMENT DESIGN}

\section{A. Data and Platform}

In order to adequately and objectively evaluate the resolution performance of the reconstructed data sets in comparison 
to the original when processed using the block-CS algorithm, we chose to perform testing on band 35 of a copy of the Indian Pines Hyperspectral Image data set [11], commonly used in hyperspectral image processing quality assessment, and band 140 of the Enrique Reef Hyperspectral Image data set [12], shown in Fig. 2. The specific bands used for this study were selected for their visual contrast between the main elements of the image. The Indian Pines Hyperspectral Image data

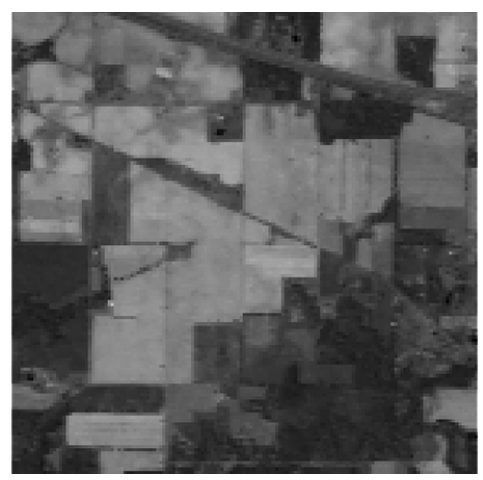

(a) Indian Pines Hyperspectral Image data set, band 35 .

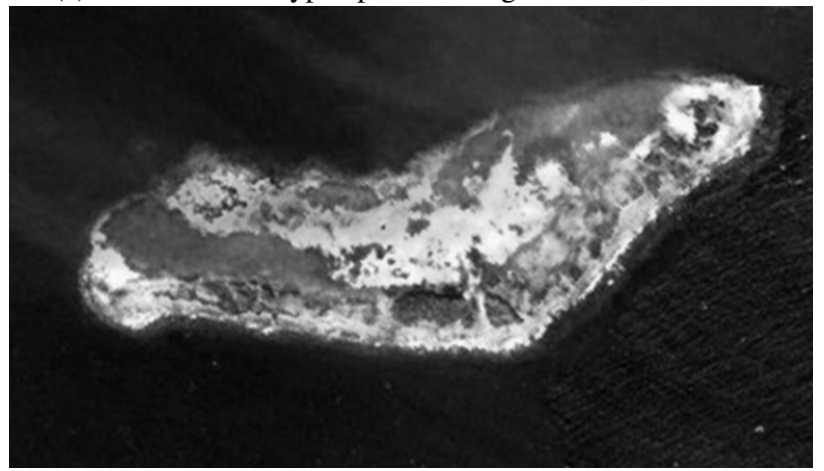

(b) Enrique Reef Hyperspectral Image data set, band 140.

Fig. 2: Selected bands from the Indian Pines and Enrique Reef Hyperspectral Image data sets.

set was captured using the Airborne/Visible Infrared Imaging Spectrometer (AVIRIS) sensor over the Indian Pines test site in northwestern Indiana, Illinois. The dataset consists of 224 spectral reflectance bands of $145 \times 145 \mathrm{px}$ each, in the wavelength range 0.4 to $2.5 \mu \mathrm{m}$. We reduced the number of bands to 200 by removing bands covering regions of high water absorption: 104-108, 150-163, and 220 [13]. This corrected dataset is the one that will be used for the proposed study. The Enrique Reef Hyperspectral Image data set, generated at the University of Puerto Rico, has been extensively studied and characterized for hyperspectral image processing algorithms [14] [15] [16].

For reference, the block-CS reconstruction algorithm procedure was executed in MATLAB on a personal computer equipped with an AMD-FX $83504.02 \mathrm{GHz}$ eight-core processor and $16 \mathrm{~GB}$ of RAM.

\section{B. Procedure}

To study the impact of compressive sampling parameters on reconstruction quality, we perform reconstructions at four different block sizes $(8 \times 8 \mathrm{px}, 10 \times 10 \mathrm{px}, 16 \times 16 \mathrm{px}$ and $32 \times 32 \mathrm{px})$ and four different sampling factors $(10 \%, 25 \%$, $50 \%$ and $75 \%$ ), for a total of 16 individual reconstructions for each of the considered data sets. In order to provide an objective metric of image reconstruction quality compared to the original images, the Structural Similarity (SSIM) index is calculated and presented for all reconstructed images [17]. The SSIM index is defined as

$$
S S I M=\frac{\left(2 \mu_{x} \mu_{y}+C_{1}\right)\left(2 \sigma_{x y}+C_{2}\right)}{\left(\mu_{x}^{2}+\mu_{y}^{2}+C_{1}\right)\left(\sigma_{x}+\sigma_{y}+C_{2}\right)},
$$

where $\left(\mu_{x}, \mu_{y}\right)$ are the image means, $\left(\sigma_{x}, \sigma_{y}\right)$ are the image variances, $\sigma_{x} y$ is the covariance of the two images, and $C_{1}, C_{2}$ are small constants that provide stability when the denominator of Equation 4 is close to zero. Here, a value close to 1 indicates a high degree of structural similarity between the two images. Conversely, a value closer to 0 indicates the images have a low structural similarity. Additional to this, we present intensity profiles of linear areas of interest with both high and low-frequency intensity variations, so as to highlight any potential degradation in image resolution due to the reconstruction process from sub-Nyquist sampling across all considered block size and sampling factor combinations. The areas of interest in both datasets are shown in Figs. 3 and 4.

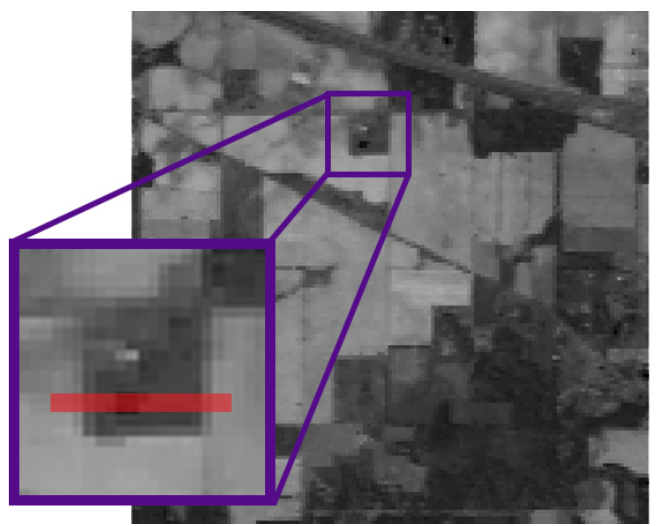

Fig. 3: Area of interest for the selected spectral band on the Indian Pines dataset, where intensity profiles will be compared across all performed reconstructions. The area of interest is highlighted in red.

\section{RESUlts}

\section{A. Indian Pines Dataset}

Fig. 5 shows a visual comparison of the reconstruction results for the Indian Pines Dataset at four different sampling factors. These results clearly show that for this image, even at a sampling factor of $10 \%$ of the original samples, the significant edge degradation and pixelation effects introduced by the subsampling process still preserve the main features of the 


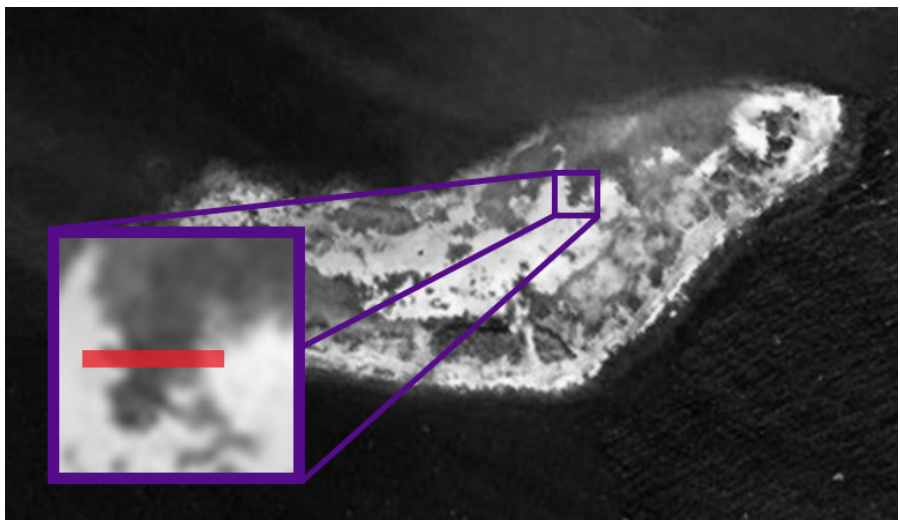

Fig. 4: Area of interest for the selected spectral band on the Enrique Reef dataset, where intensity profiles will be compared across all performed reconstructions. The area of interest is highlighted in red.

image, although fine details elude a successful recovery. Tables II and I show the reconstruction times and SSIM indexes, respectively, for all considered block size and sampling factor combinations in the Indian Pines dataset. Finally, Fig. 7 shows the intensity profiles indicated in Fig. 3. These intensity profiles show the detail with which edges are preserved under different sampling parameters when reconstructing the image from a smaller amount of samples.

TABLE I: Reconstruction error, quantified using the Structural Similarity Index (as given by Equation 4), for the Indian Pines dataset, showing all considered block size and sampling factor combinations.

\begin{tabular}{|c|c|c|c|c|c|}
\hline & \multicolumn{4}{|c|}{ Indian Pines } \\
\hline & & \multicolumn{4}{|c|}{ Sampling Factor } \\
\hline & & $10 \%$ & $25 \%$ & $50 \%$ & $75 \%$ \\
\hline \multirow{4}{*}{ Block Size } & 8 & 0.476 & 0.742 & 0.843 & 0.945 \\
\hline & 10 & 0.496 & 0.724 & 0.911 & 0.962 \\
\hline & 16 & 0.568 & 0.754 & 0.885 & 0.957 \\
\hline & 32 & 0.561 & 0.785 & 0.908 & 0.974 \\
\hline
\end{tabular}

TABLE II: Reconstruction time, in seconds, for the Indian Pines dataset, showing all considered block size and sampling factor combinations.

\begin{tabular}{c|c|cccc|}
\cline { 3 - 5 } \multicolumn{2}{c}{} & \multicolumn{4}{c|}{ Indian Pine } \\
\cline { 3 - 6 } \multicolumn{2}{c}{} & \multicolumn{4}{c}{ Sampling Factor } \\
\hline \multirow{3}{*}{ Block Size } & $\mathbf{8}$ & 34.119 & 43.302 & $\mathbf{5 0 \%}$ & $\mathbf{1 5 \%}$ \\
& $\mathbf{1 0}$ & 37.691 & 56.718 & 79.168 & 80.566 \\
& $\mathbf{1 6}$ & 79.680 & 101.197 & 155.917 & 23.001 \\
& $\mathbf{3 2}$ & 413.042 & 530.756 & 726.092 & 1040.499 \\
\hline
\end{tabular}

\section{B. Enrique Reef Dataset}

Similarly, Fig. 6 shows a visual comparison of the reconstruction results for a spectral band at three different sampling factors. Similar to the Indian Pines case, the results show that, even at a sampling factor of $10 \%$ of the original samples, the significant edge degradation introduced by the process still preserves our ability to identify the overall geographical structure in the image, although some finer details elude a successful recovery. Fig. 8 presents the profile of the area shown in Fig. 4 for all combinations of block sizes and sampling factors. Further, Tables III and IV show the SSIM indexes and reconstruction times, respectively, for all considered block size and sampling factor combinations.
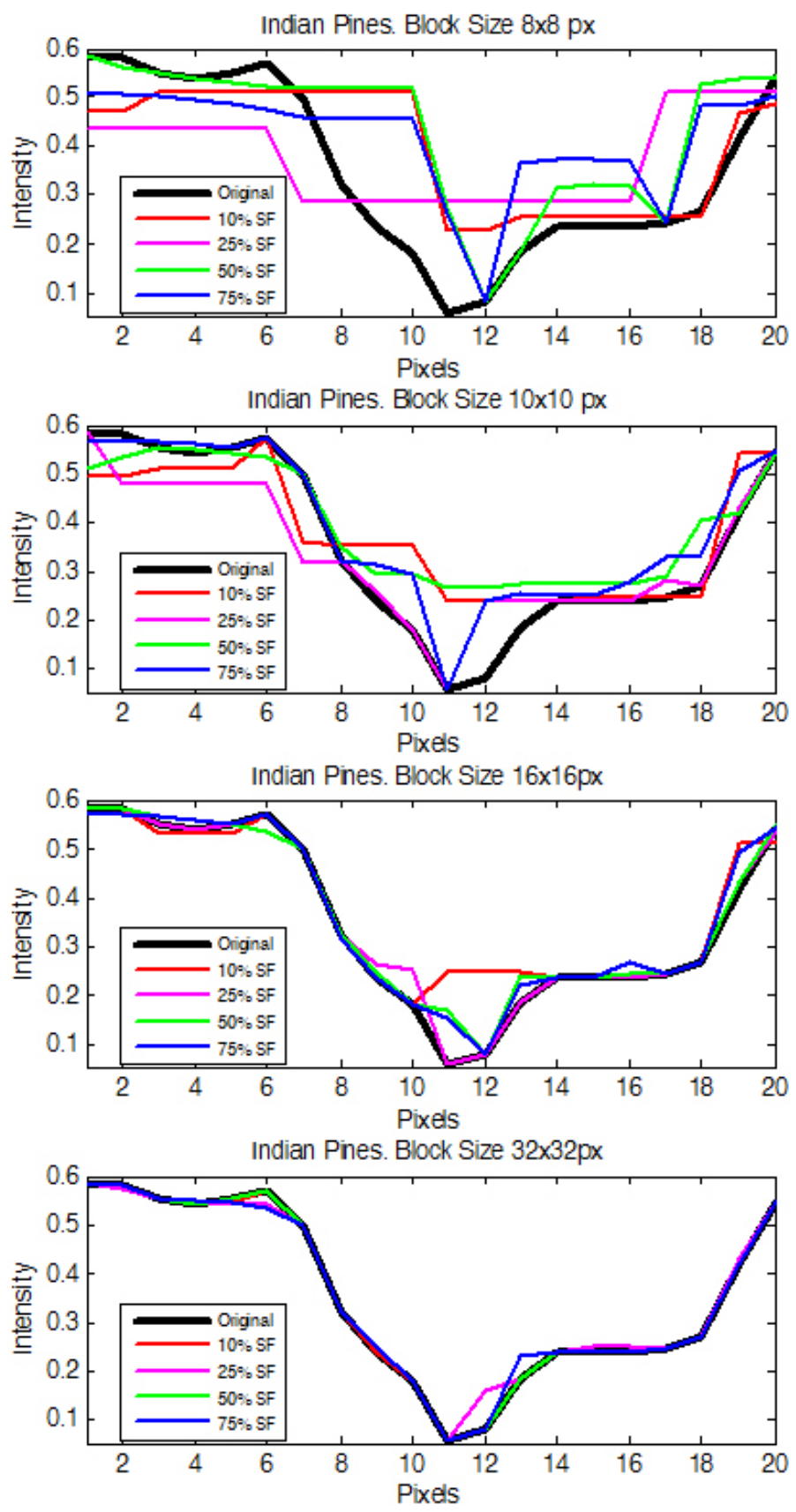

Fig. 7: Intensity profiles for the sections specified in Fig. 4 across all reconstructions of the Indian Pines dataset. 

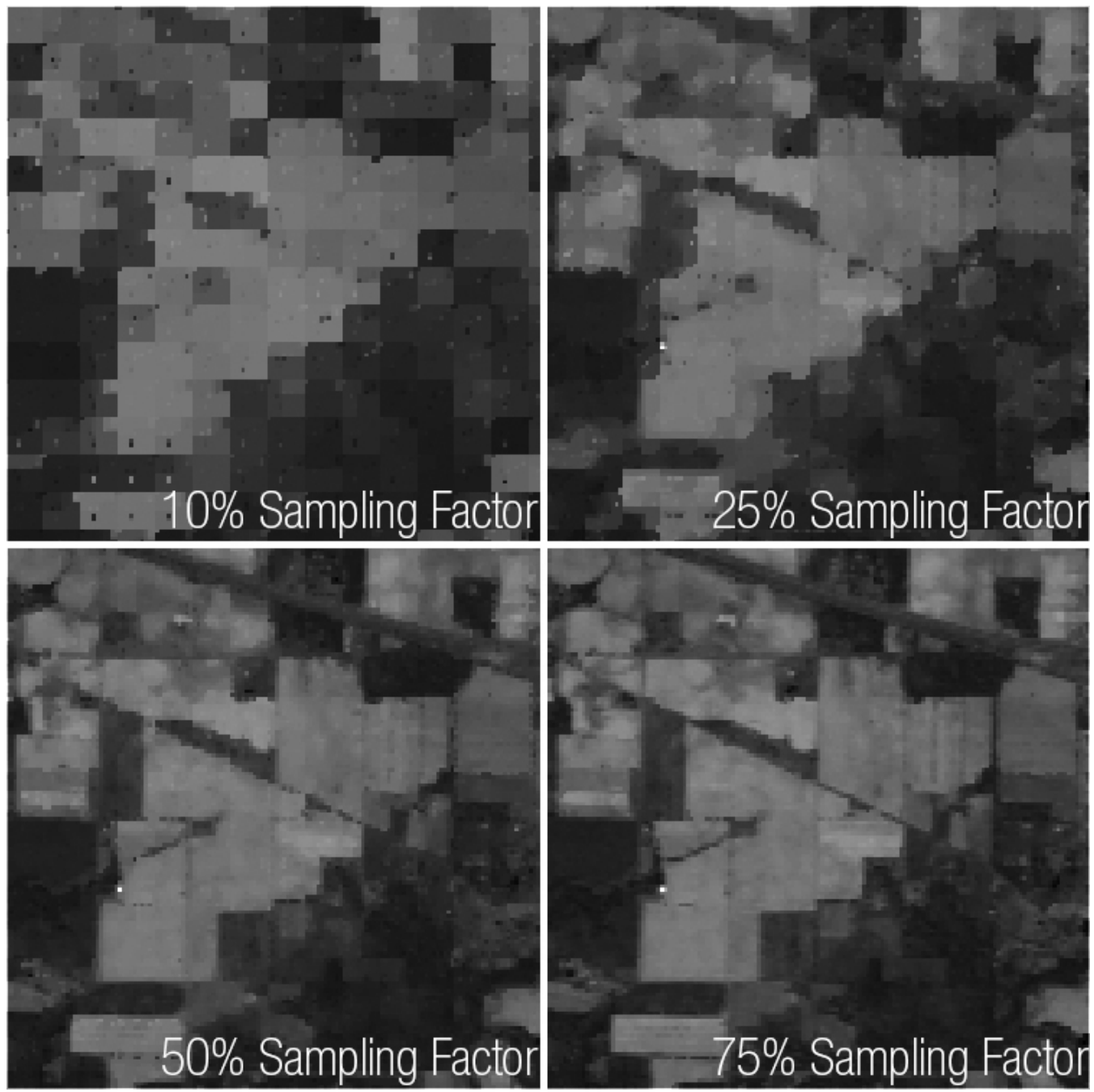

Fig. 5: Subset of reconstructed image results for the Indian Pines dataset. From left to right: Reconstruction using a 10\% sampling factor, $25 \%$ sampling factor, $50 \%$ sampling factor and $75 \%$ sampling factor. All reconstructions shown were executed using a block size of $10 \times 10$ pixels. Notice the improvement in pixel degradation proportional to the sampling factor.

TABLE III: Reconstruction error, quantified using the Structural Similarity Index (as given by Equation 4), for the Enrique Reef dataset, showing all considered block size and sampling factor combinations.

\begin{tabular}{c|c|cccc|}
\multicolumn{2}{c}{} & \multicolumn{4}{c|}{ Enrique Reef } \\
\cline { 3 - 6 } \multicolumn{2}{c|}{} & \multicolumn{4}{c|}{ Sampling Factor } \\
\hline \multirow{3}{*}{ Block Size } & $\mathbf{8}$ & 0.778 & 0.875 & 0.955 & 0.983 \\
& $\mathbf{1 0}$ & 0.789 & 0.897 & 0.956 & 0.986 \\
& $\mathbf{1 6}$ & 0.801 & 0.892 & 0.956 & 0.986 \\
& $\mathbf{3 2}$ & 0.816 & 0.905 & 0.958 & 0.986 \\
\hline
\end{tabular}

\section{Results Discussion AND CONClusion}

The results presented in Tables I and III indicate a clear, albeit not dramatic, positive correlation between block size and reconstruction quality, quantified using the Structural Similarity Index. This is a stark contrast to visual analysis of the reconstructions, which show significant edge degradation
TABLE IV: Reconstruction time, in seconds, for the Enrique Reef dataset, showing all considered block size and sampling factor combinations.

\begin{tabular}{|c|c|c|c|c|c|}
\hline & \multicolumn{4}{|c|}{ Enrique Reef } \\
\hline & & \multicolumn{4}{|c|}{ Sampling Factor } \\
\hline & & $10 \%$ & $25 \%$ & $50 \%$ & $75 \%$ \\
\hline \multirow{4}{*}{ Block Size } & 8 & 88.231 & 109.492 & 150.307 & 184.588 \\
\hline & 10 & 97.106 & 155.401 & 202.135 & 278.207 \\
\hline & 16 & 212.439 & 287.782 & 468.710 & 526.957 \\
\hline & 32 & 1161.051 & 1468.104 & 2153.506 & 2788.331 \\
\hline
\end{tabular}

and pixelation at lower block sizes, as can be observed in Figs. 7 and 8. Futher, a strong negative correlation is observable between block size and reconstruction time. As is to be expected, reconstruction quality improves with higher sampling factors. On the other hand, the required reconstruction time shows a slight increase with higher sampling factors, due to the additional data points that need to be considered in the optimization problem, although this difference is not nearly as 

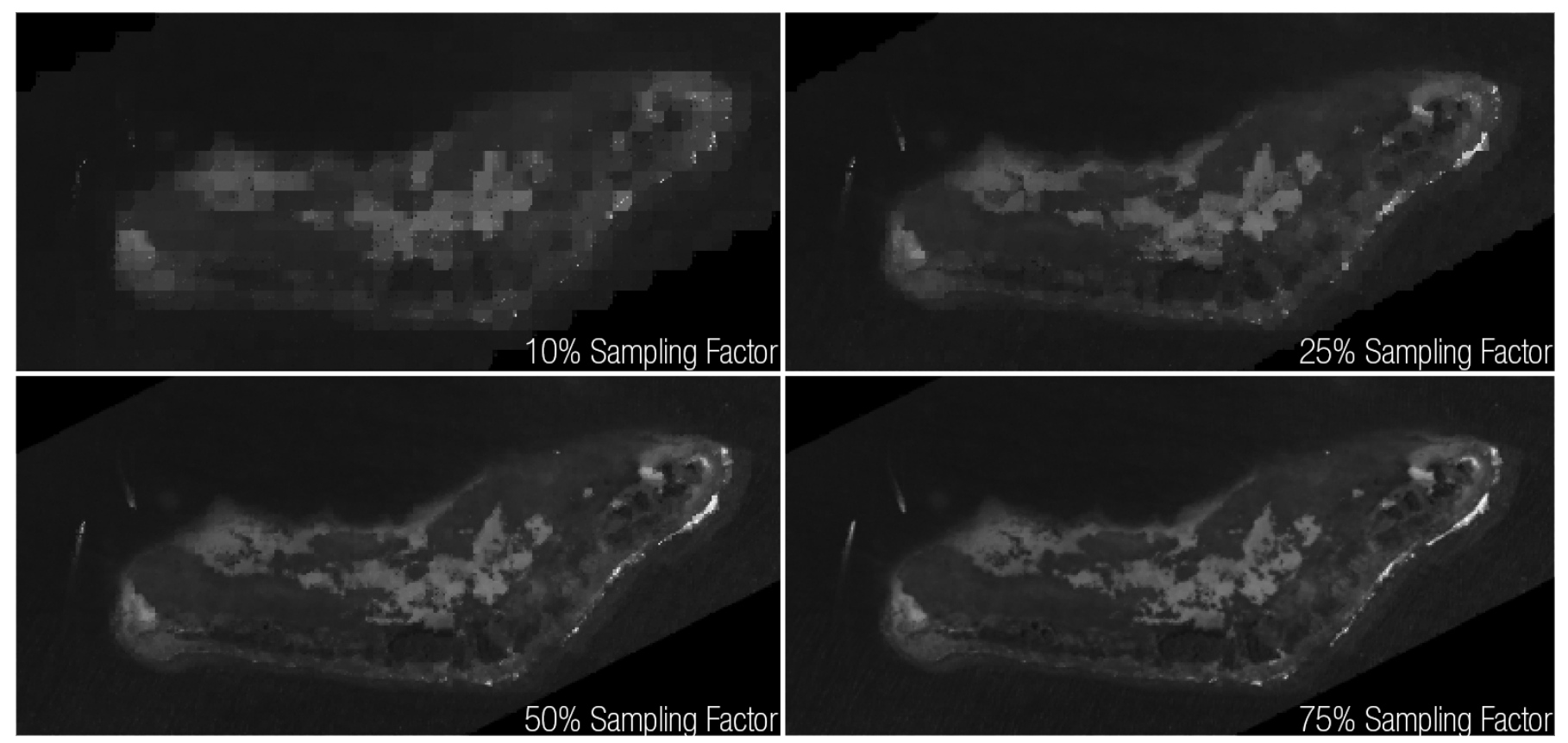

Fig. 6: Subset of reconstructed image results for a the Enrique Reef datset. From left to right: Reconstruction using a 10\% sampling factor, reconstruction using a $25 \%$ sampling factor, reconstruction using a 50\% sampling factor and reconstruction using a 75\% sampling factor. All reconstructions shown here were performed using a block size of $10 \times 10$ pixels. Notice the improvement in pixel degradation proportional to the sampling factor.

dramatic as with higher block sizes. It is important to highlight that in hyperspectral images, time of access to a sensor is a highly coveted resource, moreso than computer time. For this reason, even in situations where the total reconstruction time is larger than the acquisition time in a non-CS approach, there is a benefit present in being able to occupy the sensor in different, less time-consuming tasks. Analysis of the linear profile results indicate that larger block sizes produce, on average, reconstructions with a higher similarity to the original images, and considerably more accurate edge profiles than those produced by reconstructions using smaller block sizes. In some detection applications, this difference might appear small enough to be considered an advantage, given the extremely high reconstruction times from larger block sizes.

It is important to note that, in a significant number of the scenarios considered, sampling factors as small as $10 \%$ produce images with sufficient information to appreciate important edges and structures in the image. Further, reconstruction times for larger block sizes appear to be prohibitive in our current approach, requiring more time to solve the optimization problem than to capture the entirety of the image multiple times over using a conventional approach, while not providing a significant advantage in reconstruction quality. Visual analysis of the results clearly shows that, whenever a lower degree of precision is acceptable (such as those needed for detection cases), sampling factors as low as $10 \%$ provide a fast, adequate representation of the image structure so as to be useful for determining the need to perform further analysis on a given target. This has the potential of considerably reducing the amount of time required to acquire specific image data of interest. Due to reduced sampling times, this approach provides the ability to capture multiple images in the same amount of time it would take to capture one under a conventional approach by reducing the required usage of a hyperspectral imaging device. Future work on this subject includes evaluating the performance of the block-CS algorithm on a wider variety of hyperspectral data, evaluating classification performance of the reconstructed imagery, as well as taking advantage of parallel computing techniques to accelerate reconstruction times.

\section{ACKNOWLEDGMENTS}

F.A. is supported by a scholarship grant from the Instituto para la Formacion y Aprovechamiento de Recursos Humanos (IFARHU) office of the government of the Republic of Panama. F.A. and E.A. thank the Laboratory for Applied Remote Sensing and Image Processing at the University of Puerto Rico, Mayaguez Campus for allowing use of their facilities for the development of this project.

\section{REFERENCES}

[1] M. F. Duarte, M. A. Davenport, D. Takhar, J. N. Laska, T. Sun, K. E. Kelly, and R. G. Baraniuk, "Single-pixel imaging via compressive sampling," IEEE Signal Processing Magazine, vol. 25, no. 2, p. 83, 2008.

[2] M. Zambrano-Nunez, E. A. Marengo, and J. M. Fisher, "Coherent singledetector imaging system," in Signal Processing Systems (SIPS), 2010 IEEE Workshop on. IEEE, 2010, pp. 111-115.

[3] E. J. Candès, J. Romberg, and T. Tao, "Robust uncertainty principles: Exact signal reconstruction from highly incomplete frequency information," Information Theory, IEEE Transactions on, vol. 52, no. 2, pp. 489-509, 2006. 

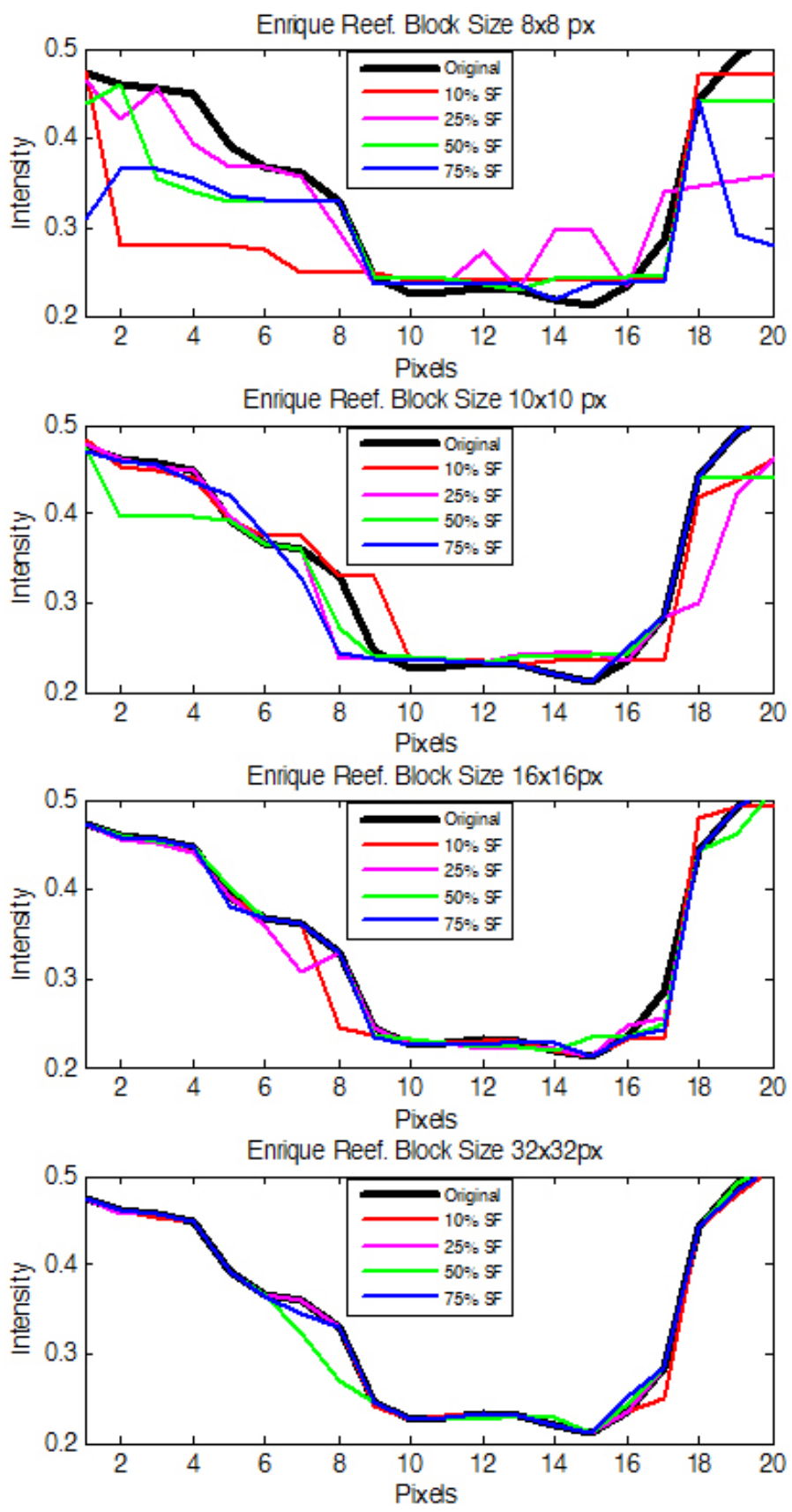

Fig. 8: Intensity profiles for the sections specified in Fig. 3 across all reconstructions of the Enrique Reef dataset.

[4] D. L. Donoho, "Compressed sensing," Information Theory, IEEE Transactions on, vol. 52, no. 4, pp. 1289-1306, 2006.

[5] M. A. Davenport, M. B. Wakin, and R. G. Baraniuk, "Detection and estimation with compressive measurements," Dept. of ECE, Rice University, Tech. Rep, 2006.

[6] G. Martin, J. M. Bioucas-Dias, and A. Plaza, "HYCA: A new technique for hyperspectral compressive sensing," IEEE Transactions on Geoscience and Remote Sensing, vol. 53, no. 5, pp. 2819-2831, 2015.

[7] A. Rajwade, D. Kittle, T.-H. Tsai, D. Brady, and L. Carin, "Coded Hyperspectral Imaging and Blind Compressive Sensing," SIAM Journal on Imaging Sciences, vol. 6, no. 2, pp. 782-812, 2013. [Online]. Available: http://epubs.siam.org/doi/abs/10.1137/120875302

[8] G. Martin, J. M. Bioucas Dias, and A. J. Plaza, "A new technique for hyperspectral compressive sensing using spectral unmixing," SPIE Optical Engineering Applications, vol. 8514, pp. $85140 \mathrm{~N}-85140 \mathrm{~N}$, 2012.

[9] M. Z. Nuñez, F. X. Arias et al., "Comparative analysis of sparse signal reconstruction. algorithms for compressed sensing." in Twelfth LACCEI Latin American and Caribbean Conference for Engineering and Technology, 2014.

[10] E. Candes and J. Romberg, "11-magic: Recovery of sparse signals via convex programming," URL: www. acm. caltech. edu/l1magic/downloads/l1magic. pdf, vol. 4, p. 14, 2005.

[11] M. F. Baumgardner, L. L. Biehl, and D. A. Landgrebe, "220 band aviris hyperspectral image data set: June 12, 1992 indian pine test site 3," Sep 2015. [Online]. Available: https://purr.purdue.edu/publications/1947/1

[12] C. Rivera-Borrero, S. Rosario, S. Hunt, C. Zayas, A. Mundorf, and S. Cardona, "Ground truth data collection for unmixing algorithm evaluation," in SPIE Defense and Security Symposium. International Society for Optics and Photonics, 2008, pp. 69661D-69 661D.

[13] J. A. Gualtieri and R. F. Cromp, "Support vector machines for hyperspectral remote sensing classification," in The 27th AIPR Workshop: Advances in Computer-Assisted Recognition. International Society for Optics and Photonics, 1999, pp. 221-232.

[14] M. C. Torres-Madronero, M. Velez-Reyes, and J. A. Goodman, "Subsurface unmixing for benthic habitat mapping using hyperspectral imagery and lidar-derived bathymetry," in SPIE Defense+ Security. International Society for Optics and Photonics, 2014, pp. 90880M-90 880M

[15] A. Santos-García and M. Vélez-Reyes, "Understanding the impact of spatial resolution in unmixing of hyperspectral images," in SPIE Defense, Security, and Sensing. International Society for Optics and Photonics, 2010, pp. 76951R-76 951R.

[16] F. N. Paravecino and V. Manian, "Spherical harmonics as a shape descriptor for hyperspectral image classification," in Proc. of SPIE Vol, vol. 7695, 2010, pp. 76 951E-1.

[17] Z. Wang, A. C. Bovik, H. R. Sheikh, and E. P. Simoncelli, "Image quality assessment: from error visibility to structural similarity," Image Processing, IEEE Transactions on, vol. 13, no. 4, pp. 600-612, 2004. 